\title{
PROFINITE GROUPS WITH RESTRICTED CENTRALIZERS
}

\author{
ANER SHALEV
}

(Communicated by Ronald M. Solomon)

\begin{abstract}
Let $G$ be a profinite group in which every centralizer $C_{G}(x) \quad(x \in$ $G)$ is either finite or of finite index. It is shown that $G$ is finite-by-abelian-byfinite. Moreover, if, in addition, $G$ is a just-infinite pro- $p$ group, then it has the structure of a $p$-adic space group whose point group is cyclic or generalized quaternion.
\end{abstract}

\section{INTRODUCTION}

The fact that free pro- $p$ groups satisfy the (ascending and descending) chain condition on centralizers indicates that, when dealing with compact groups, this condition may not be strong enough to ensure a well-behaved structure.

In this note we consider another type of restriction on centralizers, which turns out to have rather interesting consequences. Let us say that a group $G$ has restricted centralizers if every centralizer $C_{G}(x)$ of an element $x \in G$ is either finite or of finite index. On the one hand this property may be regarded as an extension of the notion of FC-groups, i.e., groups with finite conjugacy classes (cf. [10]); note that the infinite dihedral group has restricted centralizers, though it is not FC. On the other hand this condition is motivated by a recent model-theoretic work of Macpherson and Steinhorn on groups with restricted definable subsets [6]. The condition studied there implies that every definable subgroup is finite or of finite index, so in particular this holds for centralizers.

Our main result is the following.

Theorem 1.1. Let $G$ be a profinite group with restricted centralizers. Then $G$ is finite-by-abelian-by-finite.

We shall actually show that, if $\Delta$ is the FC-centre of $G$, then $G / \Delta$ and $\Delta^{\prime}$ are finite. Moreover, it will turn out that, if $G / \Delta$ is nontrivial, then it has the structure of a Frobenius complement (see Proposition 3.1). The precise structure of $G$ is determined in the special case where $G$ is a just-infinite pro- $p$ group (see Corollary 3.2).

The proof applies two powerful recent results on periodic compact groups and on centralizers in locally finite groups. The first is a theorem of Zelmanov (which extends his solution to the restricted Burnside problem), stating that

Received by the editors March 30, 1993.

1991 Mathematics Subject Classification. Primary 22C05, 20 F24. 
periodic profinite groups are locally finite [12] (see also [11]). The second result combines a theorem of Khukhro [4, p. 128] with a work of Hartley and Meixner [2] and the Classification (see [1, Theorem 1.2]); it states that a locally finite group with an automorphism of prime order and finite centralizer is nilpotentby-finite.

We remark that, by [9], a locally finite p-group with an element of finite centralizer is soluble. This result, together with a certain Lie-theoretic result of Zelmanov, enabled Zelmanov and myself to prove Theorem 1.1 in the case of pro- $p$ groups. I am grateful to Efim Zelmanov for his contribution. Subsequently, it turned out that a more direct approach may deal with profinite groups in general. I would also like to thank Avinoam Mann for several useful comments.

\section{Proof of Theorem 1.1}

Our first three lemmas deal with arbitrary groups. It is clear that the property of having restricted centralizers is inherited by subgroups but not necessarily by quotients. However, we still have:

Lemma 2.1. If $G$ has restricted centralizers and $N \triangleleft G$ is finite, then $G / N$ has restricted centralizers.

Proof. Given an element $y=x N$ of $G / N$, consider $C_{G}(x)$. If it has finite index in $G$, then $\left(G / N: C_{G / N}(y)\right)<\infty$. So suppose $C_{G}(x)$ is finite. We claim that $C_{G / N}(y)$ is finite.

Otherwise, let $H / N=C_{G / N}(y)$ and $C=C_{H}(N)$. Since $H$ is infinite and $(H: C)$ is finite, $C$ is infinite. Consider the map $f: C \rightarrow N$ sending $g$ to [ $g, x]$. Since $C$ centralizes $N, f$ is a homomorphism. Let $K=\operatorname{Ker}(f)$. $K$ is clearly infinite (as $C$ is infinite and $N$ is finite), and we have $K \subseteq C_{G}(x)$. This contradicts our assumption on $x$.

Let $\Delta=\Delta(G)$ denote the FC-centre of $G$.

Lemma 2.2. If $G$ has restricted centralizers, then $G / Z(\Delta(G))$ is periodic.

Proof. First note that every element of infinite order has infinite centralizer, so it must lie in $\Delta$. Hence all elements outside $\Delta$ are periodic, so in particular $G / \Delta$ is periodic. Finally, $\Delta / Z(\Delta)$ is periodic (as $\Delta$ is FC; see [10, p. 4]). The result follows.

Lemma 2.3. Suppose $G$ has restricted centralizers, and let $S \subseteq G$ be an infinite subset. Then $C_{G}(S) \subseteq \Delta(G)$. In particular, if $H \subseteq G$ is an infinite subgroup, then $Z(H) \subseteq \Delta(G)$.

Proof. Let $x \in C_{G}(S)$; then $S \subseteq C_{G}(x)$, so $C_{G}(x)$ is infinite and $x \in$ $\Delta(G)$.

We now turn to locally finite groups.

Lemma 2.4. Let $G$ be a locally finite group with restricted centralizers. Then either $G$ is FC or it is nilpotent-by-finite.

Proof. Suppose $G$ is not FC. Then, by Lemma $2.2, G / \Delta$ is a nontrivial periodic group. Pick an element $x \in G$ of prime order $p$ modulo $\Delta$. Let $H=C_{G}\left(x^{p}\right)$. Then $(G: H)<\infty$. Note that conjugation by $x$ induces on $H$ an automorphism of order dividing $p$ whose centralizer is finite. 
We claim that a locally finite group which admits such an automorphism is nilpotent-by-finite. Indeed, using [2] and the Classification it follows that such a group is (locally-nilpotent)-by-finite (see [1, Theorem 1.2]). The fact that the group is actually nilpotent-by-finite follows from Khukhro's result (see [3] or [4, Theorem 5.3.1, p. 128]).

Applying this result we conclude that $H$ is nilpotent-by-finite and so is $G$.

Proposition 2.5. Let $G$ be a locally finite group with restricted centralizers. Then $(G: \Delta(G))<\infty$.

Proof. We may assume that $G$ is not FC. By Lemma 2.4 there exists a nilpotent subgroup $N \triangleleft G$ with $(G: N)<\infty$. We claim that $N \subseteq \Delta$, from which the proposition follows at once.

We prove the claim by induction on $c=\operatorname{cl}(N)$, the nilpotency class of $N$. Note that $N$ is infinite (since $G$ is). Thus, if $c=1$, then $N \subseteq C_{G}(N) \subseteq \Delta$ by Lemma 2.3. Suppose $c>1$ and consider $Z(N)$. If it is infinite, then a similar argument shows that $N \subseteq C_{G}(Z(N)) \subseteq \Delta$. So assume $Z(N)$ is finite. But then $G / Z(N)$ has restricted centralizers by Lemma 1.1 , so we have $N / Z(N) \subseteq$ $\Delta(N / Z(N))$ by induction. However, $Z(N) \subseteq \Delta$ and $\Delta(G / Z(N))=\Delta(G) / Z(N)$ (since $Z(N)$ is finite). We conclude that $N \subseteq \Delta$ as required.

In order to prove the main theorem it is essential to deal first with compact FC-groups.

Lemma 2.6. Let $G$ be a profinite $F C$-group. Then $G^{\prime}$ is finite.

Proof. For $n \geq 1$ let $\Delta_{n}$ be the set of elements $x \in G$ satisfying $\left(G: C_{G}(x)\right) \leq$ $n$. It is easy to see that $\Delta_{n}$ is a closed subset of $G$; the union of all these subsets is the whole of $G$. By Baire Category Theorem there exists $n$ such that $\Delta_{n}$ contains a nonempty open subset. This means that $g H \subseteq \Delta_{n}$ for some $g \in G$ and an open normal subgroup $H \subseteq G$. By the well-known inclusion $\Delta_{n} \Delta_{m} \subseteq \Delta_{n m}$ we easily obtain $H \subseteq \Delta_{n^{2}}$. Hence $H$ is a BFC-group, so $H^{\prime}$ is finite (cf. [7]). Now $G / H^{\prime}$ is an abelian-by-finite FC-group, hence it is centralby-finite. By Schur's Theorem (cf. [10, p. 2]) it follows that $G^{\prime} / H^{\prime}$ is finite and so is $G^{\prime}$.

It follows from the above result that if $G$ is a profinite group and $\Delta(G)$ is closed in $G$, then it is finite-by-abelian. However, in general $\Delta(G)$ need not be closed: for example, if $S_{i} \quad(i \geq 1)$ are nonabelian finite simple groups and $G=\Pi S_{i}$ is their cartesian product, then $\Delta(G)$ is the direct product of the groups $S_{i}$, which is neither closed nor finite-by-abelian.

However, we shall show that, in our situation, the FC-centre $\Delta(G)$ is closed. We first deal with profinite groups which are not locally finite. This is where Zelmanov's work [12] is applied.

Lemma 2.7. Let $G$ be a profinite group with restricted centralizers. Suppose $G$ is not locally finite. Then

(1) $Z(\Delta(G))$ is not periodic.

(2) $(G: \Delta(G))<\infty$.

(3) $\Delta(G)$ is closed.

(4) $\left|\Delta(G)^{\prime}\right|<\infty$.

Proof. By [12] periodic profinite groups are locally finite. It follows that $G$ is not periodic. But $G / Z(\Delta(G))$ is periodic (by Lemma 2.2). We conclude that 
$Z(\Delta)$ is not periodic. This proves part (1). For parts (2) and (3), let $x \in Z(\Delta)$ be an element of infinite order. Then $C_{G}(x) \supseteq \Delta$ and $\left(G: C_{G}(x)\right)<\infty$. On the other hand, Lemma 2.3 shows that $C_{G}(x)=C_{G}(\langle x\rangle) \subseteq \Delta$. It follows that $\Delta=C_{G}(x)$ and thus $(G: \Delta)<\infty$; moreover, since centralizers in profinite groups are always closed, we conclude that $\Delta$ is closed. Finally, part (4) follows form part (3) (using Lemma 2.6).

In order to prove Theorem 1.1 it remains to deal with locally finite groups.

Proposition 2.8. Let $G$ be a profinite group with restricted centralizers. Suppose $G$ is locally finite. Then $\Delta(G)$ is closed. Consequently $\Delta(G)^{\prime}$ is finite and $G$ is finite-by-abelian-by-finite.

Proof. By Proposition 2.5 we have $(G: \Delta)<\infty$. By Lemmas 2.4 and $2.6 G$ is nilpotent-by-finite. We may assume that $G$ is infinite. Let $N \subseteq \Delta$ be a nilpotent normal subgroup of finite index in $G$. We will show by induction on $c=c l(N)$ that $\Delta$ is closed (hence open). It suffices to show that the image of $\Delta$ in some profinite image $G / C$ of $G$ is closed, where $C$ is a closed normal subgroup contained in $\Delta$.

If $c=1$, we take $C=C_{G}(N)$. Clearly $N \subseteq C \subseteq \Delta$ (by Lemma 2.3) and $C$ is closed (as centralizers are closed). Since $G / C$ is finite, hence discrete, the image of $\Delta$ is closed there, so $\Delta$ is closed. Now suppose $c>1$. If $Z(N)$ is infinite, then a similar argument works with $C=C_{G}(Z(N))$. If $Z(N)$ is finite, then it is certainly closed and $G / Z(N)$ has restricted centralizers (by Lemma 2.1). Set $C=Z(N)$, and apply the induction hypothesis to conclude that $\Delta / C=\Delta(G / C)$ is closed in $G / C$.

Therefore, $\Delta$ is closed, hence profinite, so $\Delta^{\prime}$ is finite by Lemma 2.6. The result follows.

Theorem 1.1 now follows from Lemma 2.7 and Proposition 2.8.

\section{CONCLUDING OBSERVATIONS}

In this section we discuss the structure of profinite groups with restricted centralizers in more detail. The first result provides some information on the finite group $G / \Delta$.

Proposition 3.1. Let $G$ be a profinite group with restricted centralizers. If $G$ is not an $F C$-group, then $G / \Delta(G)$ has the structure of a Frobenius complement.

Proof. Note that $\Delta\left(G / \Delta^{\prime}\right)=\Delta / \Delta^{\prime}$ and that $G / \Delta^{\prime}$ has restricted centralizers. This allows us to factor out $\Delta^{\prime}$ and assume that $\Delta$ is abelian. Suppose $G$ is not an FC-group, and let $H=G / \Delta$. Then $H$ is a nontrivial finite group and $\Delta$ is infinite.

Observe that $H$ acts on $\Delta$ in such a way that $\left|C_{\Delta}(h)\right|<\infty$ for all $1 \neq h \in H$. In order to show that $H$ has the structure of a Frobenius complement it suffices to show that $H$ acts fixed-point-freely on some finite elementary abelian group $E$ (i.e., $C_{E}(h)=1$ for all $\left.1 \neq h \in H\right)$.

Case I: $G$ is locally finite. Then $\Delta$ is a periodic abelian profinite group, so it has finite exponent. We may therefore choose a prime $p$ such that the characteristic subgroup $\Delta_{p}$ of elements of order dividing $p$ is infinite. Let $V=\Delta_{p}$. We may regard $V$ as an infinite-dimensional linear space over $\mathbb{F}_{p}$, acted on by $H$. Obviously, nonidentity elements of $H$ have finite centralizers in $V$. 
Claim 1. The order of $H$ is prime to $p$.

For otherwise, let $h \in H$ be of order $p$. For $0 \leq i \leq p$ let $V_{i}=(h-1)^{i} V$. Then $V=V_{0} \supseteq V_{1} \supseteq \cdots \supseteq V_{p}=0$. We show, by reverse induction on $i$, that $\operatorname{dim}\left(V_{i}\right)<\infty$. The case $i=p$ is clear. Now, given $i<p$ consider the epimorphism $h-1: V_{i} \rightarrow V_{i+1}$. Its kernel is finite dimensional (as $C_{V}(h)$ is finite) and its image is finite dimensional by induction. Therefore, $\operatorname{dim}\left(V_{i}\right)<\infty$ as required.

In particular, we obtain $\operatorname{dim}(V)=\operatorname{dim}\left(V_{0}\right)<\infty$. This contradiction completes the proof of the claim.

Claim 2. $H$ acts fixed-point-freely on some finite submodule $E \subset V$.

Indeed, since $p$ does not divide the order of $H, V$ is a completely reducible $\mathbb{F}_{p} H$-module. Therefore, we may write $V=\prod_{j \in J} M_{j}$ where each $M_{j}$ is irreducible (hence finite) and the index set $J$ is infinite.

Fix a nonidentity element $h \in H$. Since $C_{V}(h)$ is finite, $h$ acts fixed-pointfreely on $M_{j}$ for all but finitely many $j \in J$. Since $H$ is finite, we conclude that, for all but finitely many indices $j \in J, H$ acts fixed-point-freely on $M_{j}$. In particular, $H$ acts fixed-point-freely on some finite submodule of $V$, proving the claim.

It is now clear that $H$ has the structure of a Frobenius complement.

Case II: $G$ is not locally finite. By Lemma 2.7 there exists an element $x \in \Delta$ of infinite order. Let $B=\langle x\rangle^{H}$, the $H$-invariant (abstract) subgroup of $\Delta$ generated by $x$. Then $B$ is a finitely generated abelian group. Let $T$ be its torsion subgroup. It is easy to see that $H$ acts fixed-point-freely on the torsionfree abelian group $A=B / T$. Indeed, if $b^{h}=b t$ for some $1 \neq h \in H, t \in T$, and $b \in B \backslash T$, then $h$ centralizes $b^{k}$ where $k$ is the order of $t$. This implies that $C_{B}(h)$ (and hence $C_{\Delta}(h)$ ) is infinite, a contradiction.

Finally, since $H$ acts fixed-point-freely on a finitely generated torsion-free abelian group $A$, it has the structure of a Frobenius complement (indeed, $H$ acts fixed-point-freely on almost all elementary abelian characteristic quotients of $A$ ).

For a detailed description of the structure of Frobenius complements, see Chapter 3 of [8].

We now consider a few examples. The first one shows that the restriction given in Proposition 3.1 is in fact the only restriction on the structure of $G / \Delta$.

Example 1. Let $H$ be a Frobenius complement. Then $H$ acts fixed-point-freely on some finite elementary abelian $p$-group $E$. Let $A$ be the cartesian product of countably many copies of $E$, and let $H$ act on $A$ diagonally. If $G$ is the split extension of $A$ by $H$, then $G$ is a profinite group with restricted centralizers satisfying $G / \Delta(G)=G / A \cong H$.

Example 2. Let $N$ be a finite nonabelian nilpotent group with a fixed-point-free automorphism $\alpha$ of prime order. Let $A$ be the direct product of countably many copies of $N$, and let $\alpha$ act on $A$ diagonally. Then the split extension $G$ of $A$ by $\langle\alpha\rangle$ is a locally finite group with restricted centralizers which is neither FC nor finite-by-abelian-by-finite. Note that the nilpotency class of $\Delta(G)=A$ may be arbitrarily large.

Our next example is not locally finite. 
Example 3. Let $G$ be a $p$-adic space group with a cyclic point group; that is, $G$ is an extension of a finitely generated free $p$-adic module by a cyclic $p$-group acting faithfully and irreducibly (see [5] for background and basic properties). Then $G$ is a pro- $p$ group with restricted centralizers. The pro- 2 completion of the infinite dihedral group is of this type.

Our final result shows that the above example is in fact rather typical. Recall that a profinite group is said to be just-infinite if it is infinite with no nontrivial closed normal subgroups of infinite index.

Corollary 3.2. Let $G$ be a just-infinite pro-p group with restricted centralizers. Then $G$ is a p-adic space group whose point group is cyclic or generalized quaternion.

Proof. First note that, by previous results, $\Delta$ is open in $G$ and $\Delta^{\prime}$ is a finite (closed) normal subgroup. Since $G$ is just-infinite, it follows that $\Delta^{\prime}=1$, so $\Delta$ is abelian. Now, pick $1 \neq x \in \Delta$ and consider its (topological) normal closure $\langle x\rangle^{G}$; it is (topologically) finitely generated (as $\left.(G: \Delta)<\infty\right)$ and it has finite index in $G$ and hence, in $\Delta$. It follows that $\Delta$ is (topologically) finitely generated. Now, let $x \in \Delta$ be an element of finite order. Then the closed normal subgroup generated by $x$ in $G$ is finite and hence, trivial, so $x=1$. We conclude that $\Delta$ is torsion-free.

We have shown that $\Delta$ is a finitely generated torsion-free abelian pro- $p$ group, so it is a free $p$-adic module of finite rank. It is clear that $H=G / \Delta$ acts faithfully and irreducibly on $\Delta$ (tensored with the $p$-adic field). It follows that $G$ is a $p$-adic space group whose translation group is $\Delta$ and whose point group is $H$. Finally, since $H$ is a finite $p$-group with the structure of a Frobenius complement, it must be cyclic or generalized quaternion [8, p. 194].

The result follows.

\section{REFERENCES}

1. B. Hartley, Centralizers in locally finite groups, Group Theory (Bressanone 1986), Lecture Notes in Math., vol. 1281, Springer, Berlin, 1987.

2. B. Hartley and T. Meixner, Finite soluble groups containing an element of prime order and small centralizer, Arch. Math. 36 (1981), 211-213.

3. E. I. Khukhro, Groups and Lie rings admitting almost regular automorphisms of prime order, Group Theory (Bressanone 1989), Rend. Circ. Mat. Palermo (2) Suppl. (1990).

4. E. I. Khukhro, Nilpotent groups and their automorphisms, de Gruyter, Berlin, 1993.

5. C. R. Leedham-Green and M. F. Newman, Space groups and groups of prime power order I, Arch. Math. 35 (1980), 193-202.

6. H. D. MacPherson and C. Steinhorn, Variants of O-minimality, preprint.

7. B. H. Neumann, Groups covered by permutable subsets, J. London Math. Soc. (2) 29 (1954), 236-248.

8. D. S. Passman, Permutation groups, Benjamin, New York, 1968.

9. A. Shalev, On almost fixed point free automorphisms, J. Algebra 157 (1993), 271-282.

10. M. J. Tomkinson, FC-groups, Pitman, London, 1984.

11. J. S. Wilson, On the structure of compact torsion groups, Monatsh. Math. 96 (1983), 57-66.

12. E. I. Zelmanov, On periodic compact groups, Israel J. Math. 77 (1992), 83-95.

Institute Of Mathematics, The Hebrew University, Jerusalem 91904, IsRael 